\title{
Paciente não sindrômico com múltiplos dentes supranumerários: relato de caso
}

Multiple supernumerary teeth in non-syndromic patient: case report

\author{
Bruna Raquel Ames* \\ Aléxsandra da Silva Botezeli Stolz** \\ Paolla Zellya Borges ${ }^{* * *}$
}

\section{Resumo}

Objetivo: relatar a presença de quatorze elementos supranumerários e o manejo clínico da condição em paciente sem síndrome ou doença associada. Relato de caso: paciente do gênero feminino, 29 anos de idade, leucoderma, procurou atendimento com a queixa de não ter conseguido realizar tratamento ortodôntico. Ao exame intrabucal, constatou-se tumefação em região vestibular aos elementos 35 e 45, além da presença de elementos supranumerários parcialmente erupcionados. A história médica da paciente não foi contributiva. Foram solicitados exames de imagem, a partir dos quais foi diagnosticada a presença de quatorze elementos dentais supranumerários localizados bilateralmente, tanto em maxila como mandíbula. Foram excluídas algumas síndromes. Optou-se pela extração de todos os dentes supranumerários. Considerações finais: dentes supranumerários é uma condição pouco frequente na população e ainda menos prevalente quando considerados múltiplos dentes supranumerários em pacientes não sindrômicos. Clinicamente, elementos supranumerários podem causar danos às estruturas adjacentes e o tratamento dessa condição deve ser multidisciplinar, sendo avaliada a presença ou a ausência de síndrome associada, para optar pelo melhor tratamento, que, geralmente, se baseia na exodontia dos elementos e posterior tratamento ortodôntico.

Palavras-chave: Cirurgia Bucal. Dente supranumerário. Dente impactado. Tomografia Computadorizada por Raios- $X$.

\section{Introdução}

Dentes supranumerários são dentes a mais do que o número normal na dentição decídua ou permanente, sendo duas vezes mais comuns nessa última ${ }^{1,2}$. A prevalência de dentes supranumerários em uma base populacional varia de $0,1 \%$ a $3,6 \%{ }^{3,4}$. No entanto, quando se considera "dentes supranumerários múltiplos", que significam cinco ou mais dentes supranumerários, a prevalência foi relatada como menor que $1 \%$. A hiperdontia múltipla, ou dentes supranumerários múltiplos não sindrômicos, é diagnosticada, segundo diferentes autores, pela presença de cinco ou mais dentes supranumerários $^{6-10}$ ou pela ocorrência de dentes supranumerários em mais de uma série dental ${ }^{11,12}$. Casos envolvendo um ou dois dentes supranumerários são mais frequentes em maxila anterior, seguidos da região de pré-molares mandibulares ${ }^{13}$. Quando estão presentes múltiplos dentes supranumerários (>5), o local mais comumente afetado é a região de pré-molares mandibulares ${ }^{4}$. Supranumerários únicos ocorrem em $76 \%$ a $86 \%$ dos casos, supranumerários duplos em $12 \%$ a $23 \%$ dos casos e supranumerários múltiplos, em menos de $1 \%$ dos $\operatorname{casos}^{14}$.

Dentes supranumerários múltiplos em pacientes não sindrômicos é uma condição de baixa prevalência que, oportunamente, tem sido descrita na

\footnotetext{
Cirurgiã-dentista, Curso de Odontologia, Universidade Federal de Santa Maria, Departamento de Estomatologia, Santa Maria, Rio Grande do Sul, Brasil.

Professora doutora associada, Departamento de Estomatologia da Universidade Federal de Santa Maria, Santa Maria, Rio Grande do Sul, Brasil.

Graduanda, Curso de Odontologia, Universidade Federal de Santa Maria, Santa Maria, Rio Grande do Sul, Brasil.
} 
literatura por casos isolados ou séries de casos. A ocorrência desses é mais frequentemente nos homens, apresentando a proporção masculino/feminino de $2: 1^{4}$.

A etiologia exata dos dentes supranumerários é desconhecida; porém, várias teorias têm sido sugeridas para explicar sua presença: a teoria filogenética como regressão aos antropoides cuja fórmula dental tinha mais elementos, a herança recessiva autossômica ou ligada ao cromossomo X, uma reação anormal a um episódio traumático local, fatores ambientais, dicotomia do germe dentário e a teoria da hiperatividade da lâmina dentária são os mais aceitos $^{15}$.

Dentes supranumerários podem ocorrer isoladamente, múltiplos, unilateralmente ou bilateralmente na maxila, mandíbula ou em ambos. Sua forma e tamanho podem ou não se assemelhar ao grupo de dentes do local onde eles são encontrados nas $\operatorname{arcadas}^{3,8}$. A localização mais comum para dentes supranumerários múltiplos é a região de pré-molares $(62,1 \%)$ e, em particular, que a região de pré-molares inferiores é um local característico para dentes supranumerários múltiplos em paciente não-sindrômico ${ }^{4}$.

Os dentes supranumerários podem ser assintomáticos e apenas diagnosticados casualmente no decorrer do exame radiográfico ${ }^{6,16}$. No entanto, podem ser capazes de causar distúrbios locais diferentes, como retenção do dente decíduo, erupção tardia do dente permanente, erupções ectópicas, deslocamentos dentários, cistos foliculares e outras alterações que requerem intervenção cirúrgica ou ortodôntica $^{17,18}$. Não existe uma única opção de tratamento ideal para os dentes supranumerários. O tratamento pode variar de exodontia simples para exodontia seguida de tratamento ortodôntico ${ }^{4,19}$.

É raro que a hiperdontia ocorra isoladamente; geralmente está associada a algum outro distúrbio, como fissura de lábio, fissura palatina ou síndromes - como Síndrome de Gardner, Síndrome de Down, Displasia Cleidocraniana, Síndrome de Zimerman-Laby ou Síndrome de Noonan ${ }^{20}$.

O objetivo deste estudo é relatar um caso clínico de uma paciente adulta com múltiplos dentes supranumerários não associados a síndromes ou a outras doenças, além do manejo do caso, considerado pela literatura incomum e complexo.

\section{Relato de caso}

Paciente do gênero feminino, 29 anos de idade, leucoderma, procurou atendimento com a queixa de não ter conseguido realizar tratamento ortodôntico, pois de acordo com o profissional consultado, ela possuía dentes em excesso que deveriam ser extraídos previamente ao tratamento.

Ao exame intrabucal, constatou-se tumefação em região vestibular aos elementos 35 e 45, além da presença de elementos supranumerários parcial- mente erupcionados em região lingual inferior (Figura 1). Todos os dentes da série normal estavam erupcionados e não foi constatada nenhuma outra anomalia dentária, apenas um leve apinhamento anterior superior e inferior. A história médica da paciente não foi contributiva. Foram solicitados exames de radiografia panorâmica e tomografia computadorizada Cone Beam (Figura 2, Figura 3), a partir dos quais foi diagnosticada a presença de quatorze elementos dentais supranumerários localizados bilateralmente, tanto em maxila como mandíbula. Um exame de Raio-X de tórax também foi solicitado a fim de verificar a presença de costela bífida, que pode estar associada a síndromes que apresentam dentes supranumerários. Tais exames contribuíram para a exclusão de algumas síndromes, como Síndrome de Gardner, Síndrome de Down, Displasia Cleidocraniana, Síndrome de Zimerman-Laby ou Síndrome de Noonan. A maioria dos elementos encontrava-se em região de pré-molares mandibulares e, também, em região de pré-molares em maxila. Devido à indicação de posterior tratamento ortodôntico, optou-se pela extração de todos os dentes supranumerários.

Os procedimentos cirúrgicos foram realizados sob anestesia local, divididos por quadrantes, com intervalo de 15 dias entre os quadrantes para cicatrização dos tecidos e conforto da paciente (Figura 4, Figura 5 e Figura 6). Para assepsia e antissepsia foram utilizados PVP-I (extrabucal) e Clorexidina $1 \%$ (Intrabucal). O anestésico de escolha foi Mepivacaína $2 \%$ com vasoconstritor epinefrina $0,01 \mathrm{mg} / \mathrm{ml}$. As incisões foram realizadas com lâminas de bisturi $\mathrm{n}^{\mathrm{o}} 11$ e 15 e suturadas com fio de seda 3-0.

Ao realizarem-se os procedimentos no quarto quadrante, houve parestesia do nervo mentual, uma vez que um elemento supranumerário se localizava muito próximo do nervo. A paciente fora informada previamente da possibilidade de parestesia pelo processo cirúrgico. No transoperatório não foi possível localizar um dos elementos supranumerários, o qual se encontrava entre canino e primeiro pré-molar inferiores; Optou-se, então, por um novo momento cirúrgico, no qual o elemento também não foi localizado. Em nova Tomografia Computadorizada, constatou-se que o elemento supranumerário restante encontrava-se mais próximo da cortical lingual, o que dificultaria o acesso e visualização (Figura 7). Assim, em consenso com outros profissionais, foi decidido realizar um novo acesso cirúrgico por meio de um retalho pelo lado vestibular (Figura 8). Nos dois primeiros momentos operatórios, foi prescrito Azitromicina $500 \mathrm{mg}$, um ao dia, como antibiótico, além de anti-inflamatório e analgésico. Para este último momento pós-operatório, foram prescritos anti-inflamatório e analgésico, a saber: Diclofenaco de Sódio $50 \mathrm{mg}$, 8/8h e Paracetamol $750 \mathrm{mg}, 6 / 6 \mathrm{hs}$, ambos por três dias.

Em acompanhamento, a cicatrização após sete dias não estava ideal, portanto, mantiveram-se os 
pontos da sutura por mais quatro dias e após esse período, foram removidos. Quatro dias após a remoção da sutura, a paciente relatou dor na região acessada e gosto ruim na boca. Em avaliação clínica, constatou-se infecção dos tecidos, presença de supuração e edema na região, confirmado através de uma radiografia periapical utilizando um cone de guta percha para direcionamento (Figura 9). Devido à presença da infecção, fora prescrito Ciprofloxacino $500 \mathrm{mg}$ a cada 12 horas por sete dias e solução de Clorexidina $0,12 \%$ para bochecho, porém, mesmo com a medicação, a infecção persistiu após o tratamento. Sendo assim, a medicação foi substituída para Metronidazol $400 \mathrm{mg}$ + Amoxicilina $500 \mathrm{mg}$, $8 / 8 \mathrm{hs}$, por sete dias. No período em que esteve medicada, a paciente apresentou pouca melhora na ci- catrização dos tecidos; por isso, foi realizado novo acesso cirúrgico e limpeza da região, momento em que foi identificado um ponto remanescente da sutura invaginado nos tecidos, o que provavelmente estava fazendo com que a infecção persistisse. Após oito dias do último acesso, a sutura foi removida e observou-se boa cicatrização dos tecidos, ausência de edema e de desconforto da paciente (Figura 10).

A prescrição medicamentosa para o tratamento da parestesia ocasionada pela lesão ao nervo mentual foi Citoneurim ${ }^{\circledR} 5.000$ UI. Entretanto, não houve adesão da paciente ao tratamento. Em nova revisão clínica, seis meses após, a paciente relatou relativa melhora na sensação de "anestesia persistente", referindo-se à parestesia do nervo mentual, ainda presente.

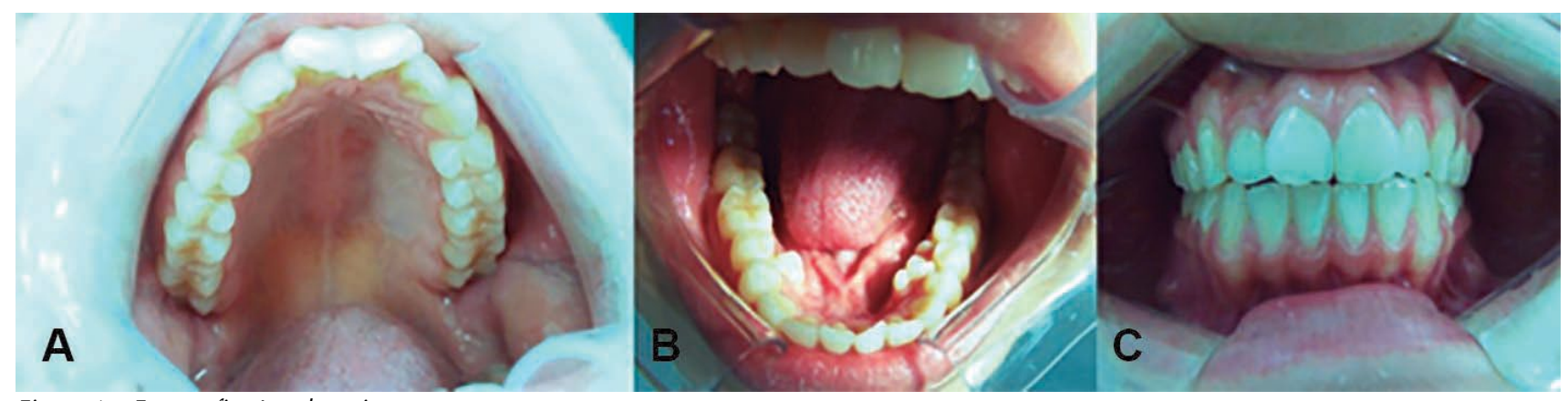

Figura 1 - Fotografias Intrabucais

Legenda: A) vista oclusal superior; B) vista oclusal inferior; C) arcadas em oclusão, presença de tumefação vestibular inferior.

Fonte: elaboração dos autores.

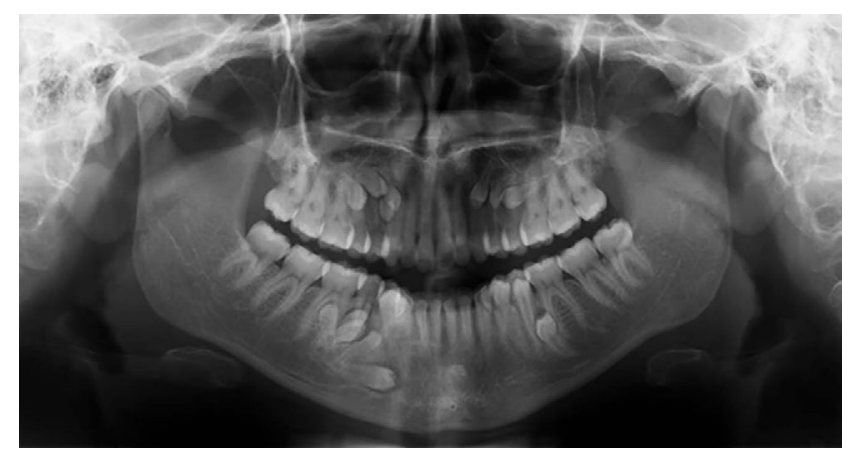

Figura 2 - Radiografia panorâmica

Fonte: elaboração dos autores.
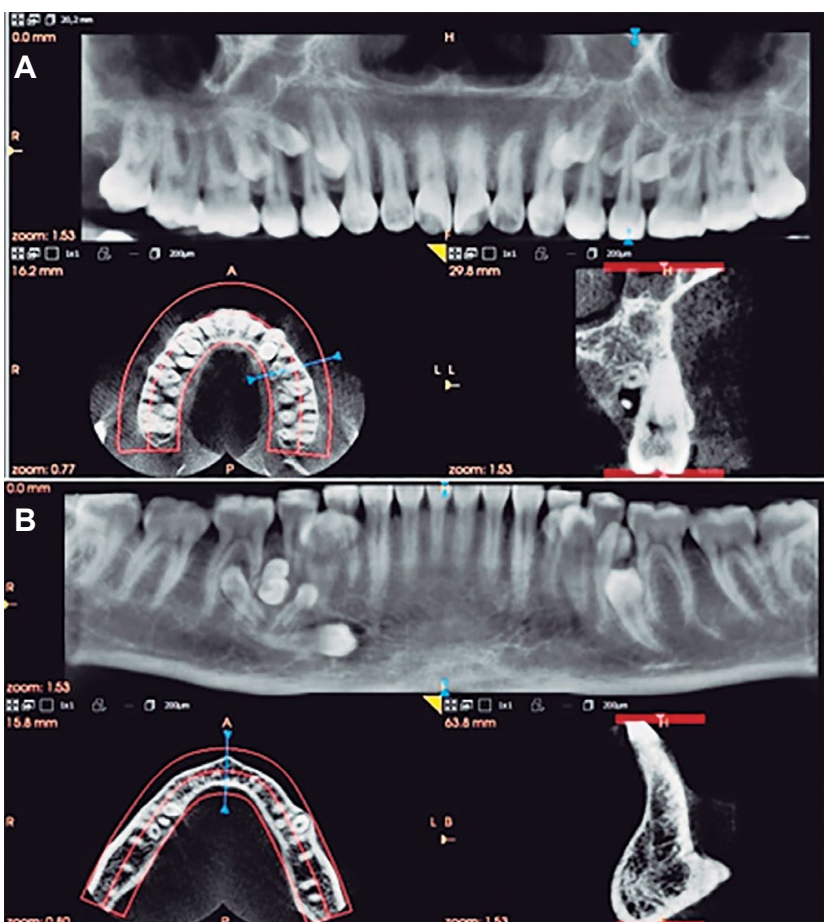

Figura 3 - Aspecto radiográfico em tomografia computadorizada Cone Beam pré-operatória

Legenda: A) cortes tomográficos de maxila; B) cortes tomográficos de mandíbula.

Fonte: elaboração dos autores. 


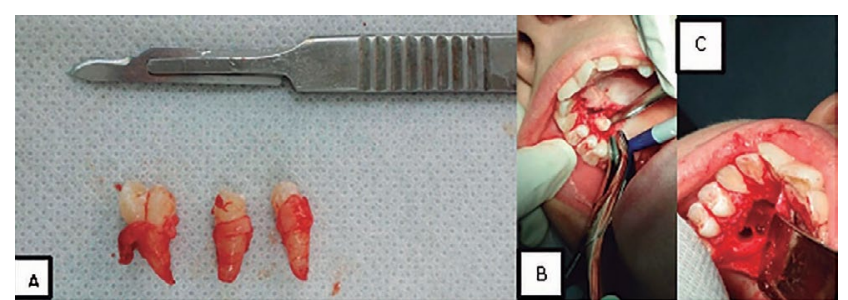

Figura 4 - Exodontia de três elementos supranumerários do primeiro quadrante

Fonte: elaboração dos autores.

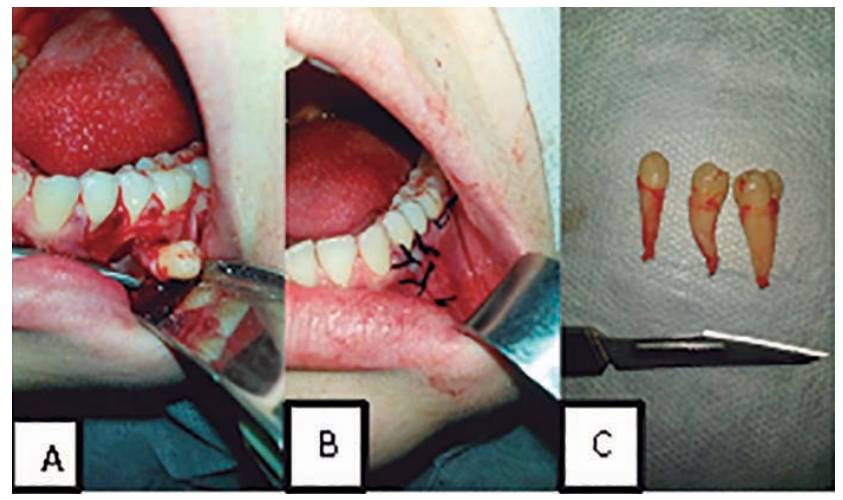

Figura 5 - Exodontia dos elementos supranumerários do terceiro quadrante

Fonte: elaboração dos autores.

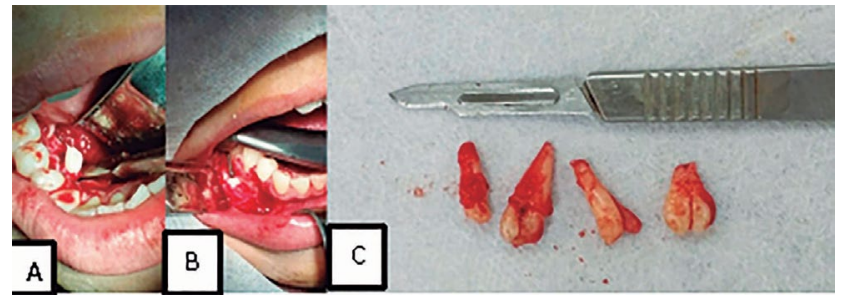

Figura 6 - Exodontia dos elementos supranumerários do quarto quadrante

Fonte: elaboração dos autores.

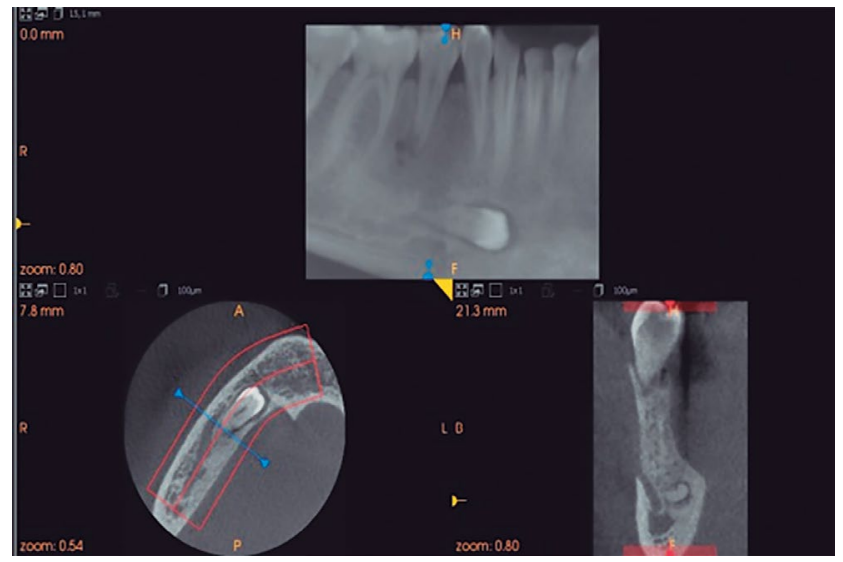

Figura 7 - Localização do elemento supranumerário em corte tomográfico e sua relação com o nervo mentual e a cortical lingual

Fonte: elaboração dos autores.

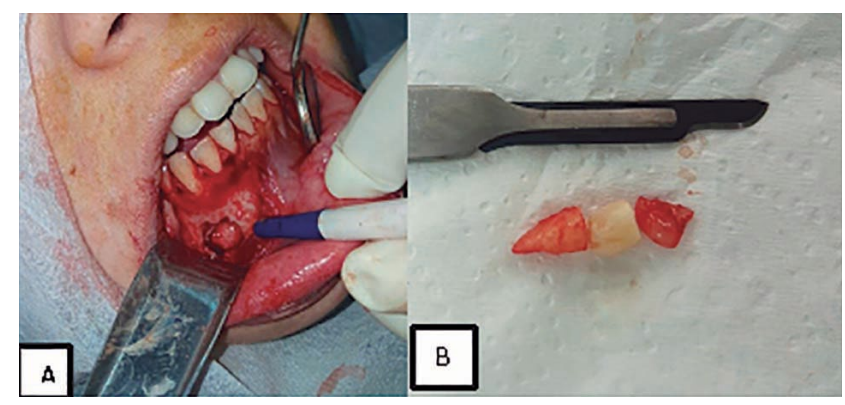

Figura 8 - Exodontia do elemento remanescente do quarto quadrante Fonte: elaboração dos autores.

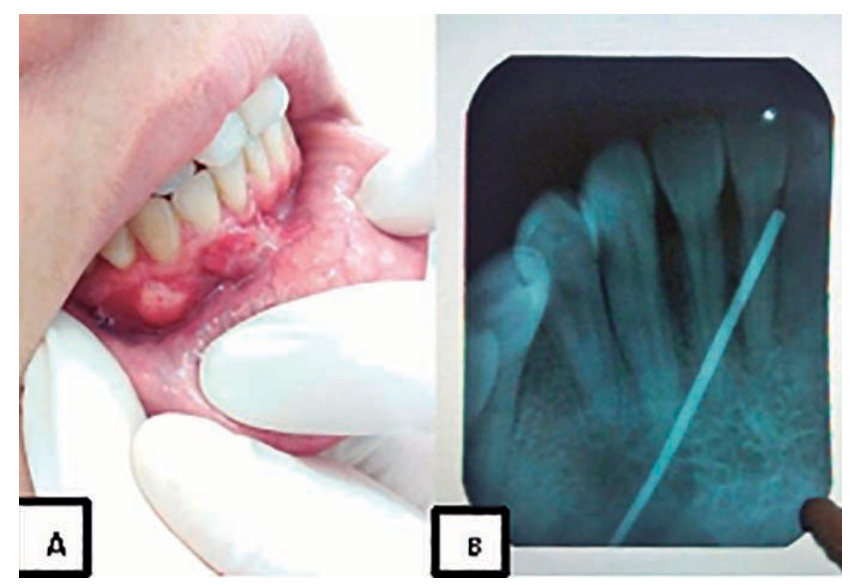

Figura 9 - Presença de infecção constatada clínica $(A)$ e radiograficamente $(B)$

Fonte: elaboração dos autores.

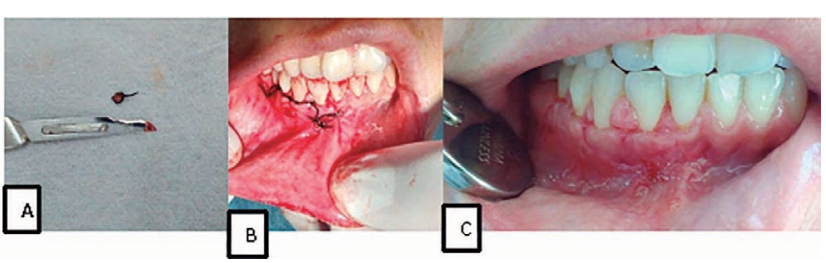

Figura 10 - Acesso para limpeza da região infectada onde foi encontrado um ponto remanescente da sutura (A), pós-operatório imediato $(B)$ e sete dias após $(C)$

Fonte: elaboração dos autores.

\section{Discussão}

Casos de pacientes não sindrômicos, como relatado no presente trabalho, apresentando múltiplos dentes supranumerários, são relativamente raros na prática clínica. Usualmente, são descobertos em radiografias e exames de rotina. Em um estudo retrospectivo realizado com 9550 pacientes, dos quais 251 tinham dentes supranumerários, apenas seis casos eram de múltiplos elementos, sendo a prevalência encontrada de $0,06 \%^{7}$. No mesmo estudo, também foi verificado que todos os pacientes afetados por múltiplos dentes supranumerários eram do gênero masculino, ao contrário do presente caso. Em outra pesquisa realizada em base de dados, foi verificado que a taxa de ocorrência de dentes supranumerários em homem: mulher foi de $2,14: 1^{19}$. Uma revisão da literatura realizada entre 1969 e 1990 
concluiu que a condição de hiperdontia múltipla é mais comum em homens do que em mulheres, em uma proporção de 9:2.

Há a singularidade de encontrar pacientes não sindrômicos com cinco, sete, oito e nove dentes supranumerários. $\mathrm{O}$ presente relato de caso trata de um paciente do gênero feminino, não sindrômico, portador de quatorze elementos supranumerários. A partir da anamnese, não foi constatada nenhuma patologia que poderia estar associada à presença dos múltiplos elementos supranumerários. A fim de confirmar a ausência dessa relação, o laudo do exame radiográfico da região de tórax trouxe o resultado negativo para a condição de costela bífida, presente em algumas síndromes associadas a múltiplos elementos supranumerários. De acordo com a literatura, síndromes que podem estar relacionadas a dentes supranumerários incluem Síndrome de Gardner, Disostose Cleidocranial e fenda de lábio e palato $^{19}$.

A exata etiologia dos dentes supranumerários permanece desconhecida, porém, várias teorias têm sido sugeridas para explicar sua presença. As mais aceitas são a atividade anormal da lâmina dentária, o que resulta na formação de dentes adicionais ou divisão dos elementos já existentes ${ }^{21}$; a possibilidade de um dente supranumerário surgir de aglomerados de epitélio remanescente após a ruptura da lâmina dentária e ser ativada a formação de dentes (teoria da dicotomia do germe dentário) ${ }^{22}$; e ainda a associação da hereditariedade também tem sido sugerida, pelo fato de a maioria dos casos serem determinados pela herança multifatorial $^{23}$. A etiologia dos dentes supranumerários é multifatorial, sendo uma combinação de fatores ambientais e genéticos ${ }^{15}$.

O tratamento dessa condição deve ser multidisciplinar, pois, segundo vários autores ${ }^{6,15,24-26}$, caso esses elementos não sejam extraídos, podem causar danos às estruturas adjacentes, como reabsorção radicular de outros dentes, erupção tardia ou falta de erupção do dente permanente, erupção ectópica, deslocamento do dente permanente, rotação do dente adjacente, formação de cistos, entre outros. As patologias mais frequentemente relatadas são atraso ou falha na erupção e má-formação do dente adjacente $^{27-29}$. No presente caso, não foi observado nenhum desses danos.

A literatura descreve diferentes opções de manejo para pacientes com hiperdontia múltipla não associada a síndromes complexas. O tratamento é parcialmente dependente da posição e manifestações clínicas do dente supranumerário. Assim, um diagnóstico precoce é muito importante para decidir entre exodontia, exodontia seguida de tratamento ortodôntico, ou simplesmente monitoramento ou controle dos dentes supranumerários, visando minimizar o risco de complicações secundárias à presença desses. Geralmente, a opção de tratamento mais utilizada consiste na remoção cirúrgica dos elementos supranumerários, seguida, muitas vezes, de tratamento ortodôntico - em torno de $62,0 \%{ }^{11}$ Nesse caso, os elementos supranumerários estavam causando apenas leve apinhamento dentário; no entanto, e em contraste com a abordagem de outros autores ${ }^{29-33}$, a remoção profilática de todos os dentes supranumerários foi decidida para evitar possíveis complicações, uma vez que tais dentes, quando deixados no local, podem entrar em erupção, alterar a oclusão ou dar origem a outros distúrbios já cita$\operatorname{dos}^{7,26,34-36}$.

Devido à complexidade do caso pelo grande número de elementos supranumerários, é necessário enfatizar a importância do uso da tomografia computadorizada como auxiliar no diagnóstico e tratamento. A tomografia nos fornece a localização exata de cada elemento e sua proximidade com estruturas importantes, além da certeza do estágio de desenvolvimento dos elementos. Além disso, Liu et al. ${ }^{16}$ (2007) afirmam que as imagens abrangentes em três planos fornecidos pela Tomografia Computadorizada de Cone Beam ajudam os cirurgiões a determinar a abordagem cirúrgica apropriada, identificando o dente supranumerário e reduzindo a quantidade de trauma cirúrgico nos tecidos adjacentes duros e moles. Recomendam também que a tomografia computadorizada seja utilizada rotineiramente para o tratamento de dentes supranumerários, especialmente para aqueles casos com múltiplos supranumerários, com má oclusão local ou com supranumerários de alta posição.

No presente estudo de caso, todos os dentes supranumerários já estavam com seu desenvolvimento completo, mas é importante salientar que o estágio de desenvolvimento é importante para o manejo cirúrgico dos dentes supranumerários, principalmente em região de pré-molares de mandíbula, sendo indicada a exodontia quando o desenvolvimento do elemento estiver completo ${ }^{17}$. Uma vez que existe sempre o risco de lesar alguma estrutura anatômica adjacente durante a extração, a relação risco-benefício da remoção dentária deve ser avaliada em todos os casos ${ }^{29,33,37}$.

Ao submeter o organismo a tais procedimentos, envolvendo desgaste ósseo e posterior reparação, são ativados mecanismos de defesa do sistema imune no qual uma cascata de eventos celulares e moleculares altamente integrados direciona o processo de restauração tecidual, em várias fases diferentes, porém, sobrepostas. A cicatrização defeituosa em feridas agudas e crônicas geralmente ocorre quando há falhas para avançar por meio das fases normais da reparação da ferida ${ }^{38}$. No caso apresentado, um ponto remanescente da sutura foi foco de infecção dos tecidos, prolongando o processo de cicatrização com a formação de tecido de granulação. O uso de medicamentos antibióticos é rotineiro na prática clínica, porém, observou-se que apesar da terapia medicamentosa administrada, a reação do organismo não foi suficiente para combater a infecção 
e expulsar o corpo estranho, e, por isso, o local foi acessado novamente para limpeza e debridamento, procurando auxiliar o organismo no processo de cicatrização.

Ao concluir o processo de cicatrização óssea, em torno de seis meses após o último procedimento, é possível que seja iniciado o tratamento ortodôntico a fim de corrigir desajustes oclusais, já que esse costuma ser o seguimento do tratamento da condição de múltiplos dentes supranumerários ${ }^{4,19}$, além de avaliar a cicatrização alveolar bem como possíveis complicações decorrentes dos procedimentos.

\section{Considerações finais}

Os dentes supranumerários são anomalias dentárias relativamente comuns, e sua prevalência varia de $0,1 \%$ a $3,8 \%$. O caso apresentado é raro, pois apresenta quatorze dentes supranumerários, tanto em maxila quanto em mandíbula, em paciente do sexo feminino. Na maioria das vezes, o diagnóstico é feito por meio de radiografias de rotina, como no caso apresentado. O tratamento é eminentemente cirúrgico complementado por tratamento ortodôntico. Complicações cirúrgicas devem ser resolvidas com criterioso exame clínico sendo o tratamento farmacológico adjuvante em muitos casos. Relatos de caso clínico incomum como este, servem de alerta para que cirurgiões dentistas estejam atentos ao criterioso diagnóstico e à conduta terapêutica.

\section{Abstract}

Objective: to report the presence of 14 supernumerary elements and the clinical management of the condition in a patient without associated syndrome or disease. Case Report: a 29-year-old female patient, leucoderma, sought treatment with a complaint that she had not been able to perform orthodontic treatment. At the intraoral examination, swelling was observed in the vestibular region of elements 35 and 45, in addition to the presence of partially erupted supernumerary elements. The patient's medical history was not contributory. Image exams were requested, from which the presence of fourteen supernumerary dental elements located bilaterally, both in the maxilla and the mandible, was diagnosed. Some syndromes were excluded. The extraction of all supernumerary teeth was chosen. Final considerations: supernumerary tooth is an uncommon condition in the population, and even less prevalent when considered multiple supernumerary teeth in non-syndromic patients. Clinically, supernumerary elements can cause damage to adjacent structures and the treatment of this condition should be multidisciplinary, evaluating the presence or absence of associated syndrome, to opt for the best treatment, which usually is based on the dental extraction of the elements and later orthodontic treatment.

Keywords: Surgery, Oral. Tooth, Supernumerary. Tooth, Impacted. Tomography, X-ray Computed.

\section{Referências}

1. Gorlin RJ, Goldman HM. Thoma's oral pathology. 6th edn. St Louis: C V Mosby Company; 1970.

2. Clayton JM. Congenital dental anomalies occurring in 3557 children. ASDC J Dent Child 1956; 23:206-8.

3. Scheiner MA, Sampson WJ. Supernumerary teeth: a review of the literature and four case reports. Aust Dent J 1997; 42(3):160-5.

4. Yusof WZ. Non-syndrome multiple supernumerary teeth: literature review. J Can Dent Assoc 1990; 56:147-9.

5. Manrique Mora MC, Bolanos Carmona MV, Briones Lujan MT. Molarization and development of multiple supernumerary teeth in the premolar region. J Dent Child 2004; 71:171-4.

6. Rajab LD, Hamdan MAM. Supernumerary teeth: Review of the literature and a survey of 152 cases. Int J Paediatr Dent $2002 ; 12(4): 244-54$.

7. Açikgöz A, Açikgöz G, Tunga U, Otan F. Characteristics and prevalence of non-syndrome multiple supernumerary teeth: a retrospective study. Dentomaxillofacial Radiol 2006; 35(3):185-90.

8. Batra P, Duggal R, Parkash H. Non-syndromic multiple supernumerary teeth transmitted as an autosomal dominant trait. J Oral Pathol Med 2005; 34(10):621-5.

9. Desai RS, Shah NP. Multiple supernumerary teeth in two brothers: a case report. J Oral Pathol Med 1998; 27(2):411-3.

10. Kaya GS, Yapici G, Omezli MM, Dayi E. Non-syndromic supernumerary premolars. Med Oral Patol Oral Cir Bucal $2011 ; 16: 522-5$

11. Yagüe-García J, Berini-Aytés L, Gay-Escoda C. Multiple supernumerary teeth not associated with complex syndromes: a retrospective study. Med Oral Patol Oral Cir Bucal 2009; 14-7.

12. Ferriol-Fiol MN, Xavier S, Figueiredo R, Valmaseda-Castellón E, Gay-Escoda C. Hiperodoncia múltiple no asociada a síndromes. A propósito de dos casos. Med Oral Patol Cir Bucal 2011; 16(ed. esp.):89-93.

13. Mitchell L. Supernumerary teeth. Dent Update 1989; 16:65-9.

14. So LLY. Unusual supernumerary teeth. Angle Orthod 1990; 60:289-92.

15. Rao PV, Chidzonga MM. Supernumerary teeth: literature review. Cent Afr J Med 2001; 47:22-6.

16. Liu DG, Zhang WL, Zhang ZY, Wu YT, Ma XC. Three-dimensional evaluations of supernumerary teeth using cone-beam computed tomography for 487 cases. Oral Surg Oral Med Oral Pathol Oral Radiol Endod 2007; 103(3):403-11.

17. Oliveira Gomes C De, Drummond SN, Jham BC, Abdo EN, Mesquita RA. A survey of 460 supernumerary teeth in Brazilian children and adolescents. Int J Paediatr Dent 2008; 18(2):98-106.

18. Zilberman Y, Malron M, Shteyer A. Assessment of 100 children in Jerusalem with supernumerary teeth in the premaxillary region. ASDC J Dent Child 1992; 59:44-7.

19. Alvira-González J, Gay-Escoda C. Non-syndromic multiple supernumerary teeth: Meta-analysis. J Oral Pathol Med 2012; 41(5):361-6.

20. Bayar GR, Ortakoglu K, Sencimen M. Multiple impacted teeth: report of 3 cases. Eur J Dent 2008; 2:73-8.

21. Foley MF, Del Rio CE. Supernumerary teeth: report of a case. Oral Surg Oral Med Oral Pathol 1970; 30(7):60-3. 
22. Saarenmaa L. The origin of supernumerary teeth. Acta Odontol Scand 1950; 9:293-303.

23. Farmer ED, Lawson FE. Stones oral and dental diseases. 5 th ed. Edinburgh and London: E\&S Livingstone Ltd; 1966.

24. Leco Berrocal MI, Martín Morales JF, Martínez González JM. An observational study of the frequency of supernumerary teeth in a population of 2000 patients. Med oral Patol oral Cir bucal 2007; 12(2):134-8.

25. Mopager V, Sudha P, Anegundi RT, Kulkarni S, Tavarageri A. Supplemental premolars in a 13 year old child - a case report. J Indian Soc Pedod Prev Dent 2002; 20:169-72.

26. Mason C, Rule DC, Hopper C. Multiple supernumeraries: the importance of clinical and radiographic follow-up. Dentomaxillofac Radiol 1996; 25:109-13.

27. Asaumi JI, Shibata Y, Yanagi Y, Hisatomi M, Matsuzaki H, Konouchi H, et al. Radiographic examination of mesiodens and their associated complications. Dentomaxillofac Radiol 2004; 33:125-7.

28. Nazif MM, Ruffalo RC, Zullo T. Impacted supernumerary teeth: a survey of 50 cases. J Am Dent Assoc 1983; 106(2):201-4.

29. Hopcraft M. Multiple supernumerary teeth. Case report. Aust Dent J 1998; 43(1):17-9.

30. GündüzK,MuglaliM.Non-syndromemultiplesupernumerary teeth: a case report. J Contemp Dent Pract 2007; 8:81-7.

31. Kalra N, Chaudhary S, Sanghi S. Non-syndrome multiple supplemental supernumerary teeth. J Indian Soc Pedod Prev Dent 2005; 23:46-8.

32. Orhan A, Ozer L, Orhan K. Familial occurence of nonsyndro-mal multiple supernumerary teeth. A rare condition. Angle Orthod 2006; 76:891-7.

33. Cochrane SM, Clark JR, Hunt NP. Late developing supernumerary teeth in the mandible. Br J Orthod 1997; 24:293-6.

34. Fernández Montenegro P, Valmaseda Castellón E, Berini Aytés L, Gay Escoda C. Estudio retrospectivo de 145 dientes supernumerarios. Med Oral Patol Oral Cir Bucal 2006; 11:339-44.

35. Gay Escoda C, Mateos Micas M, España Tost A, Gargallo Albiol J. Otras inclusiones dentarias. Mesiodens y otros dientes supernumerarios. Dientes temporales supernumerarios. Dientes temporales incluidos. En: Gay Escoda C, Berini Aytés L, editores. Tratado de Cirugía Bucal. Tomo I. Madrid: Ergon; 2004. p. 497-534.

36. Arathi R, Ashwini R. Supernumerary teeth: a case report. J Indian Soc Pedod Prev Dent 2005; 23:103-5.

37. Nayak UA, Mathian VM, Veerakumar. Non-syndrome associated multiple supernumerary teeth: a report of two cases. J Indian Soc Pedod Prev Dent 2006; 24:11-4.

38. Morethson, P. Farmacologia para a Clínica Odontológica/ Priscilla Morethson. Coordenador da série Oswaldo Crivello Junior. Rio de Janeiro: Santos; 2015.

Endereço para correspondência:

Bruna Raquel Ames

Universidade Federal de Santa Maria

Rua Floriano Peixoto, 1184/215

CEP: 97015-372 Santa Maria, RS, Brasi

E-mail: bruna_ames@hotmail.com paolla.borges@gmail.com

Recebido: 24/10/17. Aceito: 05/12/17. 